\title{
Spontaneous Pneumomediastinum, Pneumothoraces and Surgical Emphysema in a COVID-19 Patient
}

\author{
Dr Sonam Tshering ${ }^{* 1}$, Dr Saquib Navid Siddiqui ${ }^{2}$, Dr Osman Ahmed ${ }^{3}$, Dr Sangay Phuntsho ${ }^{4}$ \\ ${ }^{1}$ Internal Medicine Trainee, Southend University Hospital, United Kingdom \\ ${ }^{2}$ Medical Registrar, East Kent Hospital, United Kingdom \\ ${ }^{3}$ Medical Registrar, Southend University Hospital, United Kingdom \\ ${ }^{4}$ Medical Officer, Phuentsholing District Hospital, Bhutan \\ *Corresponding author: Dr Sonam Tshering; sonamtshering56@gmail.com
}

Received 03 February 2021; $\quad$ Accepted 15 February 2021;

Published 24 February 2021

\begin{abstract}
Pneumomediastinum is defined as the presence of air or other gas in the mediastinum and is also known as mediastinal emphysema Pneumomediastinum can be categorized as spontaneous (SPM) or traumatic. Traumatic pneumomediastinum is caused by blunt or penetrating trauma to the chest, or iatrogenic injury, such as that produced by thoracic surgery. Mechanical ventilation is a common cause of barotrauma and pneumomediastinum. Pneumomediastinum caused by mechanical ventilation is often considered a type of traumatic pneumomediastinum, rather than SPM (UpToDate).

We report a case of spontaneous pneumomediastinum, pneumothorax and surgical emphysema in a 20-year-old patient with COVID-19. He was managed conservatively.
\end{abstract}

Keywords: Spontaneous pneumomediastinum (SPM), Pneumothorax, Surgical emphysema, COVID-19, Alveolar rupture.

\section{Background}

SPM is uncommon with reported incidence ranging from 1 in 800 to 1 in 42,000 in adult and paediatric patients admitted to a hospital. (UpToDate)

SPM occurs when air leaks through small alveolar ruptures to the surrounding bronchovascular sheath ${ }^{[2]}$. Less commonly, pneumomediastinum results from air escaping from the upper respiratory tract, intrathoracic airways, or gastrointestinal tract (eg, esophageal perforation) ${ }^{[3]}$. The common predisposing factors for SPM are asthma exacerbations and lower respiratory tract infections. Less common triggers include Valsalva manoeuvre, cough, vomiting, oesophageal rupture, measles, choking, and barotrauma (UpToDate).

Because the mean pressure in the mediastinum is always more negative than the pressure in the pulmonary parenchyma, the free air tends to move centripetally along the vascular sheaths, perhaps facilitated by the pumping action of breathing. The air dissects to the hilum and spreads into the mediastinum or through the loose mediastinal fascia to the subcutaneous tissues of the thorax, upper limbs, and neck (UpToDate).

Very rarely, pressure accumulates in the mediastinal cavity, causing pneumothorax or compression of adjacent intrathoracic structures (tension pneumomediastinum or tension pneumopericardium). These complications are probably triggered by an abrupt rise in mediastinal pressure ${ }^{[4]}$.

Air may also leak into the spinal canal, a phenomenon known as pneumorrhachis. This may have associated neurologic signs, which are usually mild and self-limited ${ }^{[5]}$.

The diagnosis is confirmed by chest imaging such as chest $\mathrm{X}$ ray and $\mathrm{CT}$ chest. The management is usually conservative with analgesics and oxygen. However, for tension pneumomediastinum, limited mediastinotomy may be performed to drain the pneumomediastinum ${ }^{[6]}$.

\section{Description}

A 20-year-old male with no medical background history, nonsmoker, presented with four-day history of central, pleuritic chest pain (worse on inspiration). He also had cough, fever and body aches for about one week duration. He subsequently tested positive for COVID 19. He did not report any breathing difficulty. No history of vomiting, abdominal pain, limb weakness or substance abuse.

On examination, he was clinically stable with oxygen saturation of $99 \%$ on room air, Pulse of 90 BPM and normal blood pressure. The chest was clear to auscultation. 
Bloods showed normal CRP, WCC, Troponin and D-dimer. Electrocardiogram showed normal sinus rhythm.
CXR (Fig 1) showed pneumomediastinum, bilateral apical small pneumothoraces and small surgical emphysema over the left upper chest.

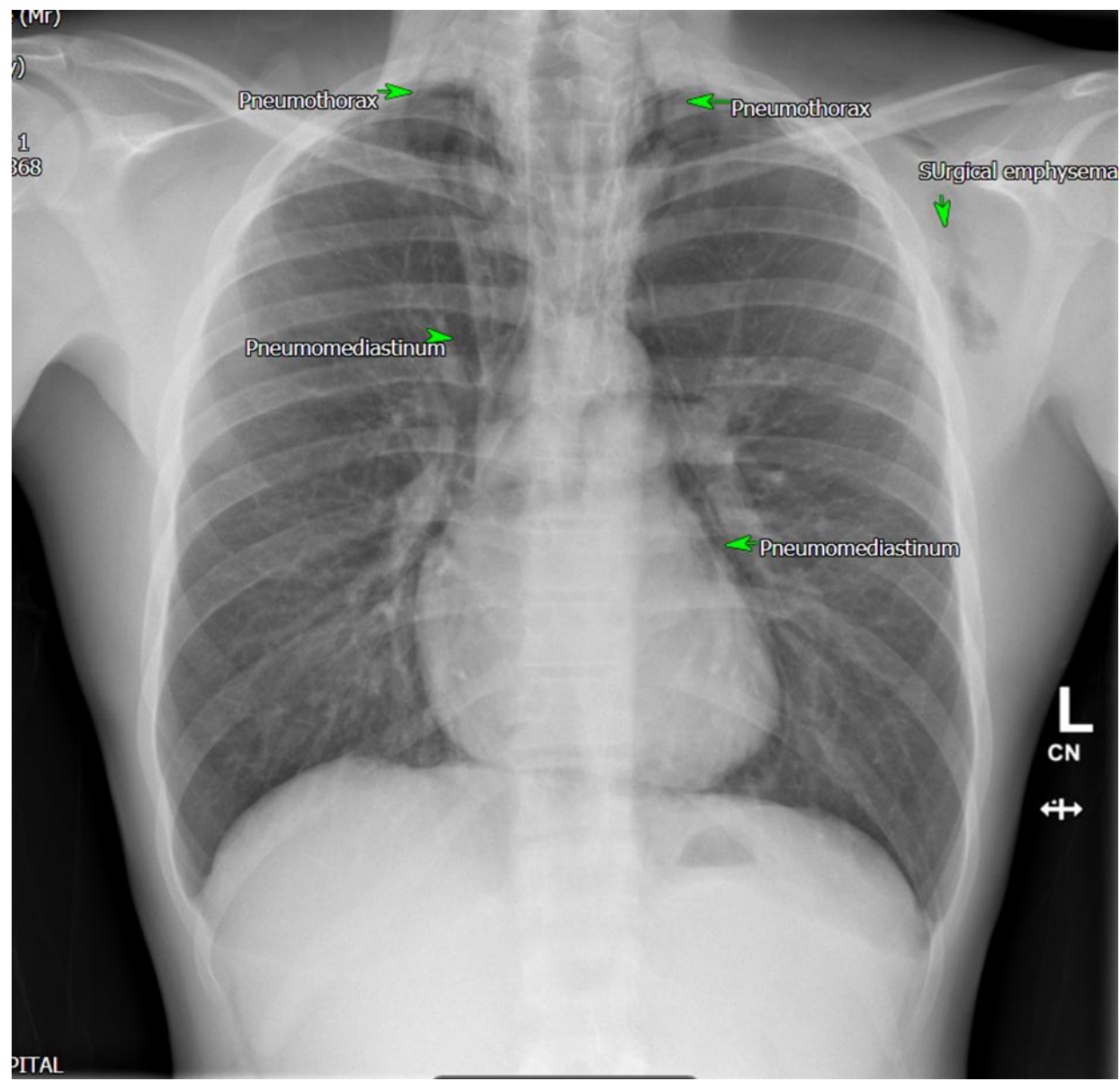

Fig 1: CXR showing pneumomediastinum, bilateral pneumothoraces, surgical emphysema.

Subsequent CT neck and chest (Fig 2) confirmed extensive pneumomediastinum, small bilateral pneumothoraces and soft tissue emphysema which extended from the neck to the lower chest wall. It was interesting to note that the lung parenchyma was normal without any ground glass changes. It also showed gas locules within the posterior spinal canal.

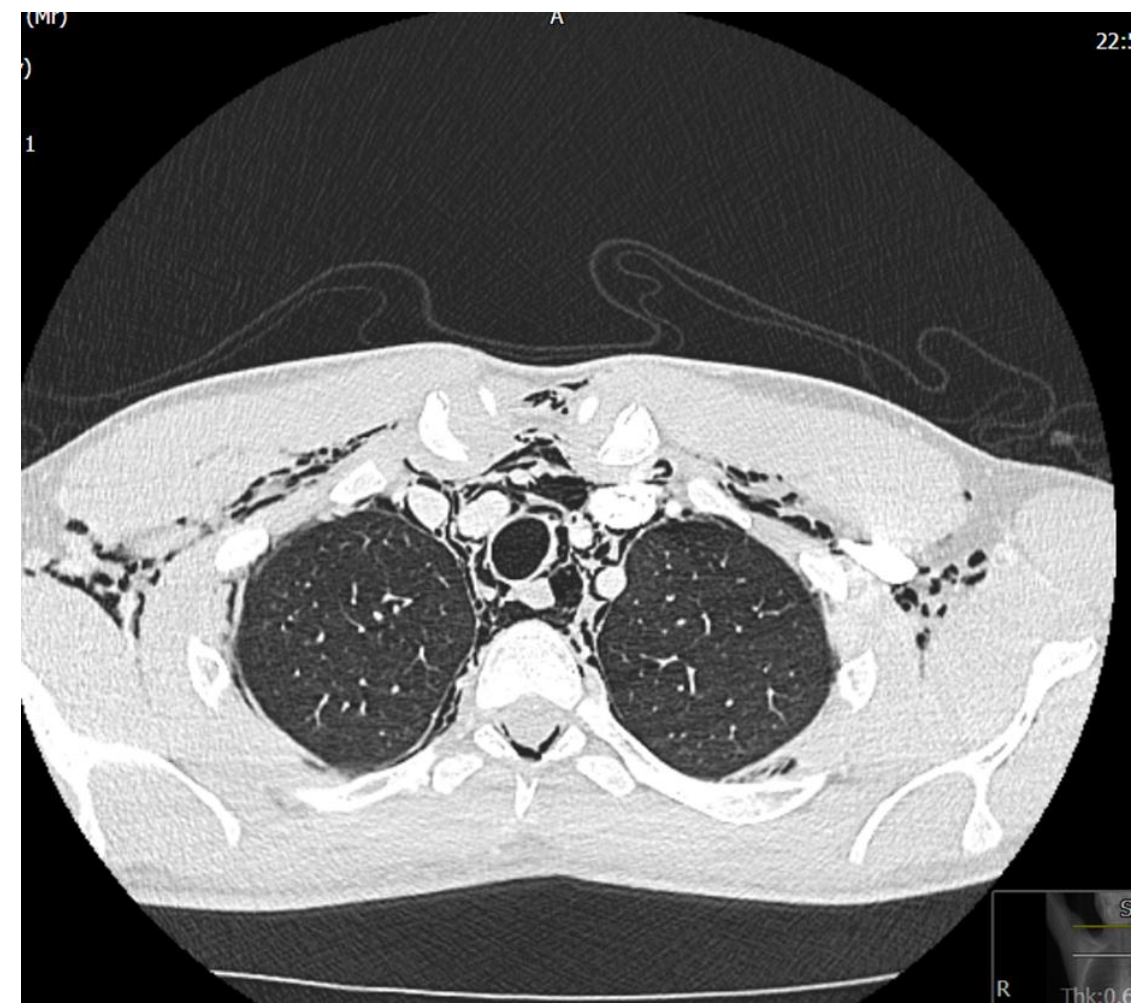

Fig 2: CT chest showing pneumomediastinum and surgical emphysema. 
There was no history of trauma nor did he receive any mechanical or non-invasive ventilation to explain pneumomediastinum.

He was managed conservatively with analgesics and discharged home with a plan for outpatient Chest $\mathrm{X}$ ray in two weeks' time.

\section{Learning points}

- Pneumomediastinum, pneumothoraces, and surgical emphysema are rare complications of COVID-19 19 even without receiving invasive or non-invasive ventilation.

- SPM mostly occurs when air leaks through small alveolar ruptures to the surrounding broncho-vascular sheath.

- The management for pneumomediastinum and surgical emphysemas are mainly conservative.

\section{Ethics approval and consent to participate}

Not applicable

\section{List of abbreviations}

SPM: Spontaneous pneumomediastinum

CT: Computed Tomography

CRP: C-reactive protein

WCC: White cell count.

\section{Data availability}

Not applicable.

\section{Conflict of interest}

No conflict of interests from all the authors

\section{Funding statement}

No funding required.

\section{Author's contribution}

Dr. Sonam Tshering; contributed to case history writing. Dr Saquib Navid Siddiqui; contributed to proof reading the paper. Dr Osman Ahmed; contributed to editing the paper.

Dr. Sangay Phuntsho; contributed to editing the paper.

\section{References}

[1] Mason, R. Pneumomediastinum and mediastinitis. In: Murray and Nadel's Textbook of Respiratory Medicine, 4th ed, Elsevier Health Sciences, 2005. Chapter 72

[2] Transport of air along sheaths of pulmonic blood vessels from alveoli to mediastinum: clinical implications, Macklin; CC; Arch Intern Med. 1939; 64:913.

[3] Mason, R. Pneumomediastinum and mediastinitis. In: Murray and Nadel's Textbook of Respiratory Medicine, 4th ed, Elsevier Health Sciences, 2005. Chapter 72.

[4] Isolated tension pneumopericardium in a case of acute lymphoblastic leukemia. Rao KL, Imamuddin S, Kumar AP; Indian Heart J. 2013 Dec;65(6):705-6.

[5] Air within the spinal canal in spontaneous pneumomediastinum. Belotti EA, Rizzi M, RodoniCassis P, Ragazzi M, Zanolari-Caledrerari M, Bianchetti MG; Chest. 2010;137(5):1197.

[6] Massive spontaneous subcutaneous emphysema. Acute management with infraclavicular "blow holes". Herlan DB, Landreneau RJ, Ferson PF Chest. 1992;102(2):503. 